\title{
Analysis of errors in finished DNA sequences: the surfactin operon of Bacillus subtilis as an example
}

\author{
Céline Fabret, Yves Quentin, Annick Guiseppi, Jeanine Busuttil, \\ Jacques Haiech and François Denizot
}

Laboratoire de Chimie Bactérienne, 31 Chemin Joseph Aiguier BP 71, 13277 Marseille Cedex 9, France

\author{
Author for correspondence: François Denizot. Tel: +3391 164387. Fax: +339171 8914 . \\ e-mail: denizot@lcb.cnrs-mrs.fr
}

Keywords: Bacillus subtilis, genome sequencing, accuracy, error, surfactin

\section{INTRODUCTION}

Recent developments in current DNA sequencing methodologies have produced systems with high productive capacity that have made it possible to start sequencing entire genomes of up to $100 \mathrm{Mb}$ (Wilson $e t$ al., 1994). The large amount of data obtained has to be accurate enough to produce final sequences with a minimum number of errors. Indeed, errors in DNA sequences may jeopardize some aspects of their analysis, for instance (a) in the design of specific oligonucleotides, (b) in studies of natural variations, and (c) in the detection of ORFs. The nature and distribution of errors in an individual sequence (i.e. a reading from one sequencing reaction) have been extensively studied (Chen \& Hunkapiller, 1992; Khurshid \& Beck, 1993; Koop et al., 1993; Sulston et al., 1992). The total error rate was shown to depend on the methodology used and on the length of reading. It is almost constant $(<1 \%)$ for readings not exceeding 350 bases, but it then increases very steeply, reaching more than $25 \%$ for readings longer than 550

Abbreviation: indel, insertion/deletion.

The EMBL accession number for the nucleotide sequence data reported in this paper is $X 72672$. bases (Koop et al., 1993). This high level of error at one extremity of the sequence may be troublesome when assembling sequences in a shotgun approach. Errors in finished sequences have not been studied so precisely, and only estimates of the error rate in DNA sequences have been made, in two databases. They vary from 0.03 to $0.3 \%$ in GenBank (Krawetz, 1989) to up to $3 \%$ in the EMBL sequence database (Kristensen $e$ t al., 1992). This is too high a level of error to do any of the above-mentioned analyses. As a matter of fact, the accuracy of a sequence can be greatly improved when increasing the depth of coverage (Churchill \& Waterman, 1992), but if the sequencing conditions are not changed, only the number of random errors will be reduced.

Before becoming participants in the $B$. subtilis genome sequencing project, we were subcontracted to the $A$. Danchin group (Institut Pasteur, Paris) and received a $\lambda$ clone containing a $13.7 \mathrm{~kb}$ DNA fragment for sequencing. It appeared to correspond to a part of the surfactin operon, sfr $A$, located at $29^{\circ}$ on the $B$. subtilis genetic map (Anagnostopoulos et al., 1993). This locus has also been determined by two other groups (Fuma et al., 1993; Cosmina et al., 1993). The three sequences, obtained independently by the three groups, overlap by more than $10 \mathrm{~kb}$ and offer the opportunity to analyse sequencing errors in finished sequences. In this report we present the 
results of this study and discuss the relationship observed between the nature of errors and the sequencing strategy.

\section{METHODS}

DNA manipulations and sequencing. The starting material (recombinant $\lambda$ bacteriophage Fix containing a $13.7 \mathrm{~kb}$ fragment of DNA from B. subtilis 168) was provided by P. Glaser and A. Danchin (Institut Pasteur, Paris). A shotgun approach was used first. The insert from the recombinant phage was sonicated and DNA fragments, ranging from 500 to $2000 \mathrm{bp}$, were sub-cloned in pUC18. Selected sub-clones were sequenced, and all steps, including plasmid denaturation (Zimmermann et al., 1990), sequencing reactions (Sanger et al., 1977) and in some cases loading of samples on polyacrylamide gels, were performed with a robot (De Araujo Novaes \& Denizot, 1993). We used T7 polymerase (Pharmacia) and $\left[\alpha-{ }^{35}\right.$ S $]$ dATP (Sequetide, NEN) as label. Polyacrylamide gels $(0.4 \mathrm{~mm}$ thick) were poured by the flap technique (Radola, 1980), using surface-treated glass plates (Barnett \& Davidson, 1989; Garoff \& Ansorge, 1981), and run using a saline gradient (Lang \& Burger, 1990) allowing a mean reading of 225 bases. After sequencing 300 templates, 16 contigs were obtained spanning more than $90 \%$ of the total sequence. At this stage, we searched for clones containing the longest inserts and overlapping two different contigs. This allowed us to position contigs with respect to each other and estimate the distance between them. After designing primers (OLIGo program, Rychlik \& Rhoads, 1990) for each extremity of the contigs, the gaps were filled by sequencing either the plasmids overlapping two contigs or the PCR products obtained using primers from two consecutive contigs. In the latter case, the same primers were also used for sequencing, following the protocol of Salles et al. (1992). Several other primers were defined to complete the sequence on both strands and to resolve areas presenting discrepancies. Finally, 7-deaza-dGTP (Mizusawa et al., 1986) and/or formamide gels (Howard, 1992) were used to remove ambiguous compressions. Each base was read an average of 5.5 times.

Data handling and sequence analysis. Gels were read manually using the PARROT program and a 5-key keyboard ( $T$ and $t$ Research). We used the University of Wisconsin Genetics Computer Group (UWGCG) software package (Devereux $e t a l$., 1984) for sequence assembling and analysis of the sequences was done mainly with the programs COMPARE, BESTFIT, PILEUP and STEMLOOP included in this package. Database searching was done using the BLAST program of the NCBI e-mail server (Altschul et al., 1990). Sequencing error predictions were performed with a modified version of the RECSTA program (Fichant \& Gautier, 1987).

\section{RESULTS}

\section{Strategies and sequences}

We sequenced DNA from B. subtilis 168 (Trp, surfactin non-producer), using the approach described in Methods, and obtained $13763 \mathrm{bp}$ which was shown to correspond to part of the surfactin operon. This sequence was submitted to the EMBL data base (accession number X72672).

Two other groups published sequences corresponding to the same chromosomal region. In a joint project, conducted between the University of Tsukuba, Japan, and the Louisiana State University Medical Center, Shreveport, LA, USA, 20535 bp was obtained (Fuma et al., 1993). DNA from the same strain was used $(168, \operatorname{tr} p C 2)$,

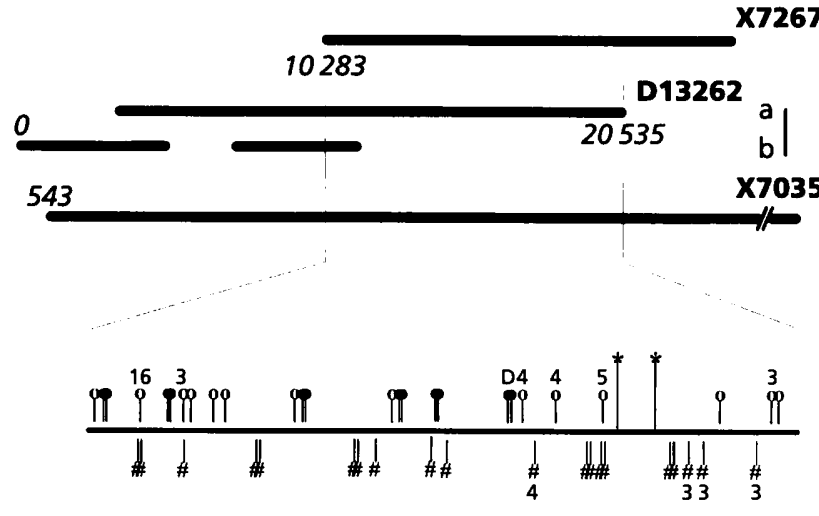

Fig. 1. Main features of the D13262, X70356 and X72672 sequences. The relative positions of the sequence are shown in the upper part of the figure using the D13262 numbering (italic type). D13262 is composed of three different sequences: the largest one (a) was obtained at the University of Tsukuba, Japan, and the two others (b) were determined at the Louisiana State University Medical Center, Shreveport, LA, USA. The lower part of the figure corresponds to a zoom of the overlapping region (10283-20535) and shows the distribution of the discrepancies found in the three sequences. Symbols 0 , * and \# correspond to discrepancies in X70356, X72672 and D13262, respectively. When several discrepancies are close together, their number is indicated either above or below the symbol. $D$ indicates the position of the duplicated area in $\times 70356$.

at least in the area we are concerned with (see Fig. 1), to obtain the sequence (GenBank name BACSFRAB, accession number D13262). This was done mainly by shotgun sequencing, the remaining gaps being filled using specific primers. An automated sequencer with fluorescent label was used for sequencing both strands by the dideoxy method. The sequencing was done under normal conditions (i.e. without using nucleotide analogues and formamide gels) (K. Yamane, personal communication). Cosmina et al. (1993), using DNA from the surfactin producer JH642srf ${ }^{+}$, obtained a $31182 \mathrm{bp}$ sequence (GenBank name BSSFAP, accession number X70356). They used the dideoxy method, mostly with a primerwalking strategy.

Throughout the text, accession numbers will be used to refer to individual sequences. Fig. 1 shows the alignment of the three sequences and their relative positions. To facilitate further analysis of these sequences, we used the numbering that corresponds to the longest sequence at the $5^{\prime}$ end, i.e. D13262. The region we selected for further investigation is the overlap between the three sequences, that is between nucleotides 10283 and 20535 .

\section{Analysis of sequence discrepancies}

We detected 86 conflicting regions between the three sequences which are the result of substitutions or insertions/deletions (indels). A duplication in X70356 (bases 16329-16345, duplicated after position 16346) was counted as a single event. The distribution of the discrepancies and their assignment to a given sequence are shown in Fig. 1. Substitutions involved a single base or a doublet. In all cases the double substitutions could be 
Table 1. Description and features of the discrepancies found between the $X 72672(1)$ and D13262 (2) sequences

\begin{tabular}{|c|c|c|c|c|}
\hline Position* & Type & $\begin{array}{c}\text { Compression } \dagger / \\
\text { stem-loop } \neq\end{array}$ & $\begin{array}{c}\text { Sequence } \\
\text { bearing error }\end{array}$ & $\begin{array}{l}\text { Corrected } \\
\text { sequence } \$\end{array}$ \\
\hline 11060 & \multirow{2}{*}{ Indel $\|$} & $-1-$ & 2 & aaačagaa \\
\hline 11062 & & $-1-$ & 2 & acagAaaaa \\
\hline 11655 & Inversion & $-1-$ & 2 & tcagCGccaa \\
\hline 12750 & \multirow{2}{*}{ Indel } & $+/+^{a}$ & 2 & cggaCgccg \\
\hline 12756 & & $+/+^{a}$ & 2 & ccgg'cgag \\
\hline 14134 & \multirow{2}{*}{ Indel } & $+1-$ & 2 & $\operatorname{ctgc}^{2} \operatorname{cgct}$ \\
\hline 14136 & & $+1-$ & 2 & gccgCtcac \\
\hline 14431 & Point & $-1-$ & 2 & gagaAcgaa \\
\hline 15246 & Inversion & $+1+$ & 2 & cattGTcggc \\
\hline 15469 & Inversion & $-1+$ & 2 & agtcCGctgt \\
\hline 16662 & Point & $-1-$ & 2 & acatAccga \\
\hline 16703 & \multirow{3}{*}{ Indel } & $+/+$ & 2 & gatcGgcgc \\
\hline 16738 & & $-1-$ & 2 & gccgGatgc \\
\hline 16765 & & $-9 /+$ & 2 & atcgGacgc \\
\hline 17523 & Point & $+/+^{b}$ & 2 & agttCgcca \\
\hline 17536 & Point & $+1+b$ & 2 & gcggCttat \\
\hline 17716 & Point & $+/+^{c}$ & 2 (Fig. 2) & atttCgcgc \\
\hline 17719 & Point & $+/+^{c}$ & 2 (Fig. 2) & $\operatorname{tcgcG} c c t g$ \\
\hline 17928 & Inversion & $+/+^{* *}$ & 1 & $\mathrm{cgcaGCtttg}$ \\
\hline 18482 & Inversion & $-1-$ & 1 & cggaGCtttt \\
\hline 18755 & \multirow{2}{*}{ Indel } & $-1-$ & 2 & atatGcgac \\
\hline 18770 & & $-1-$ & 2 & gttč gcaa \\
\hline 18939 & \multirow{3}{*}{ Indel } & $-1-$ & 2 & caaa`acgg \\
\hline 18946 & & $-1-$ & 2 & ggttcatc \\
\hline 19004 & & $-1-$ & 2 & atac aacg \\
\hline 19126 & \multirow{2}{*}{ Indel } & $-1-$ & 2 & cttg waaat \\
\hline 19156 & & $-1-$ & 2 & tctt' gaaa \\
\hline 19241 & Inversion & $+1-$ & 2 & cgaaGCttct \\
\hline 19933 & \multirow{2}{*}{ Indel } & $+1-$ & 2 & tagcCGgaga \\
\hline 19952 & & $+1-$ & 2 & cgggGcgcg \\
\hline 20001 & Indel & $+1+$ & 2 & aagcGGTttgt \\
\hline
\end{tabular}

* Positions refer to the numbering of sequence D13262.

f $\mathrm{A}+$ sign indicates that compressions have been detected in our sequencing gels.

$\ddagger$ The presence of a stem-loop structure is indicated with + . When the corresponding stem-loop overlaps the discrepancy, a + sign is used. Normally, the distance between a discrepancy and a stem-loop does not exceed 2 bases. Identical letters ( $a, b$ and $\mathrm{c}$ ) indicate that discrepancies correspond to the same stem-loop. $\$$ Upper-case letters indicate the corrected bases. In the case of inversions and point errors, the first uppercase letter corresponds to the indicated position. For indels, bases in upper-case letters have been inserted after the indicated position and ${ }^{v}$ means that the base at the indicated position has been deleted.

$\|$ Single events of insertion or deletion, involved in the same frameshift and preserving the reading frame, are grouped as indels.

9 No compression detected in this area, but a polymerase pause has been observed in many clones, suggesting that this region is difficult to sequence due to the presence of secondary structures.

** Unclassified stem-loop (18th position with the STEMLOop program) presenting, when enlarged with 6 bases, a stable structure.

interpreted as base inversion (i.e. CG in one sequence and $\mathrm{GC}$ in the other). Indels induce frameshifts, but no ORF interruption is observed, because each deletion (insertion) is always followed either by an insertion (deletion) or by two deletions (insertions) in the sequence. Single indel events involved in the same frameshift and preserving the reading frame were grouped. Thus, we finally distinguished three types of discrepancies: (1) single base substitutions; (2) base inversions; and (3) indels (Table 1 ). The first two types corrupted only one or two bases (and one or two amino acids), but the indel type changed as many as 24 amino acids. 
(a)

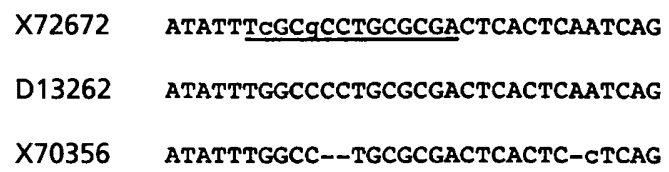

X72672 ATATTTCGCGCCTGCGCGACTCACTCAATCAG

D13262 ATATTTGGCCCCTGCGCGACTCACTCAATCAG

X70356 ATATTTG--GCCTGCGCGACTCACTC-CTCAG

(b)

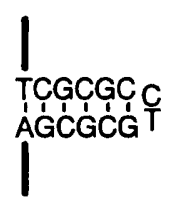

(c)

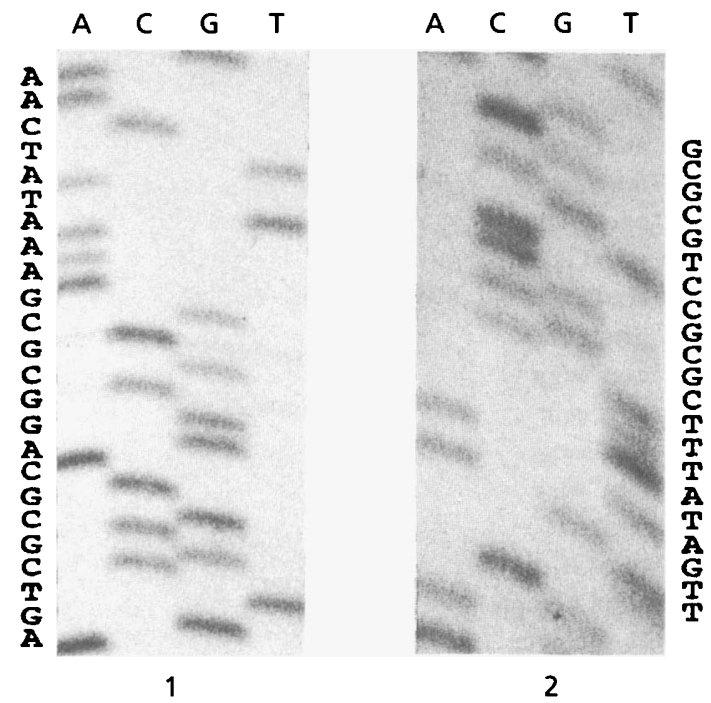

Fig. 2. Analysis of the discrepancies at positions 17716 and 17719. (a) Multi-alignments of the sequences (X72672, D13262 and $\mathrm{X70356)}$ in this region. Two different alignments are presented (depending on the position of deletions in X70356) and the discrepancies are shown in lower-case letters. (b) Stem-loop structure possibly formed by the sequence underlined in (a). (c) Autoradiographs showing the sequences obtained on both strands $(1,2)$ using 7-deaza-dGTP. Base order is indicated over the lanes and sequence readings are shown on the left (1) or right (2).

We searched for discrepancies between the X72672 and D13262 sequences and used the X70356 sequence, determined from another strain, to resolve the conflicts. As indicated in Table 1 , we found six single base changes, five GC to $C G$ inversions, one GT to TG inversion and nine indels within the 10253 bp corresponding to the overlap. After comparison with the X70356 sequence, 27 discrepancies (single event) were interpreted as sequencing errors in D13262 and two in X72672. Two other differences (17716 and 17719) cannot be resolved just by sequence alignment because a 2 bp deletion was observed in X70356 (Fig. 2). Depending on the location of the deletion in the alignments, one or two errors could be assigned to X72672. In fact, we checked our data on both strands at these positions carefully (Fig. 2), and did not find any evidence of errors in our sequence (X72672). In the end, all discrepancies were resolved and we obtained a sequence of $B$. subtilis strain 168 , which is most likely free of errors (Table 1).

In total we found 31 discrepancies. Among them, 16 correspond to areas difficult to sequence and, in most cases, to compressions detectable in our gels (compressions correspond to abnormal mobilities of DNA fragments in sequence ladders). In one instance we found polymerase pauses in almost all the templates we sequenced, suggesting that some local properties of the DNA affect the enzyme.

\section{Secondary structures and frameshifts}

It has been proposed that compressions might be caused by stretches of secondary structure in single-stranded DNA, such as stem-loops, that are stable even in the denaturating environment of a sequencing gel (Sambrook et al., 1989). We used the STEMLOop program of the GCG package (Devereux et al., 1984) to search for inverted repeats in the overlapping DNA fragments. We set the parameters to a minimum stem length of $5 \mathrm{bp}$ and a loop size between 2 and $5 \mathrm{bp}$. Among the 15 more stable stem-loops found over the $10253 \mathrm{bp}$, only seven are shared by both sequences (X72672 and D13262). The other eight hairpins are specific to X72672 and are associated with 11 discrepancies that correspond to compression artifacts in our gels (Table 1). They were all resolved and in 10 instances, X72672 appeared to be the correct sequence. This correlation between discrepancy, stable stem-loop and compression artifact, suggests that if a discrepancy is observed between two sequences at the level of a compression, the sequence giving the more stable stem-loop has the best chance to be the correct one. As an illustration, the unresolved discrepancies (17716, 17719), which were found to be associated with a stable stem-loop only in $\mathrm{X} 72672$, have been resolved as sequencing errors in D13262 (Fig. 2).

Therefore, searching for secondary structures can be used to resolve discrepancies between two sequences. One can also look for frameshift errors. This approach is particularly useful when only one sequence is available.

The distribution of the non-overlapping trinucleotides differs for each frame in coding regions and this property could be used for detecting frameshift errors. We tested this assumption by using a new algorithm based on an approach similar to the one developed in the RECSTA program (Fichant \& Gauthier, 1987) which was an algorithm devoted to the prediction of coding and noncoding sequences. We computed the trinucleotide frequencies of each frame and used the correspondence analysis method to obtain linear combinations of the trinucleotides that gave the best discrimination between the three frames. For prediction, a window was shifted along the sequence with a 30 -base step to keep in frame with the ORF. A frameshift error is detected when the 


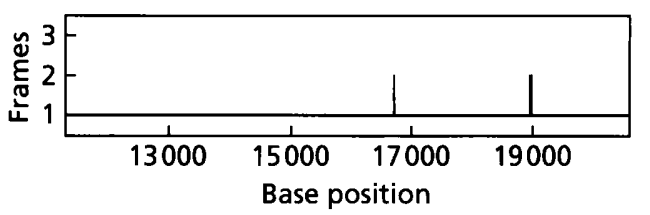

Fig. 3. Frameshift errors predicted with our program in the overlapping region. In this region (from 10283 to 20535), the first reading frame corresponds to the coding frame. A $90 \mathrm{bp}$ window was shifted along the sequence with a $30 \mathrm{bp}$ step, and assigned by the program to one of the three reading frames. Only two regions are predicted in frame 2 , and both have been described as frameshift errors of more than $60 \mathrm{bp}$ (positions 16703-16765 and 18939-19004 in Table 1).

frame of the window does not correspond to the frame of the ORF. Using a 90-base window, which corresponds to the best ratio between error predictions and false predictions, two regions of $\mathrm{D} 13262$ that should be in frame 1 were assigned to frame 2 (16703-16 765 and 18939-19004, Fig. 3). They were predicted as frameshift errors and they correspond to the larger discrepancies involving 61 and 64 bp (see grouped indels, Table 1).

We shall not describe in detail the analysis of the discrepancies observed in X70356. The sequence was obtained from a $B$. subtilis strain that produces surfactin (strain 168 does not), and at least some of the discrepancies might be due to polymorphism. However, two regions seem to correspond to sequencing errors: the duplicated area (bases 16329-16345, duplicated after position 16346) and a region where almost all Gs (16/17) are replaced with Cs (from base 10989 to base 11042). When running the modified RECSTA program with the same parameters as above, this area is predicted as a frameshift error, suggesting that the $G$ to $C$ exchanges correspond to sequencing errors. It should be pointed out that the program also predicts an error in X70356, outside the overlapping region, in position 3769-3829. It corresponds to a frameshift in this sequence. Therefore, all the frameshifts observed in X70356 and D13262, involving a large number of bases (about 60 ), were detected by the method with no false positive. These preliminary results suggest that statistical methods can be used to check new sequences for frameshift errors. This kind of error is not the most frequent $3.5 \%$ of the 86 discrepancies found between the three sequences) but certainly it is the most bothersome as it corrupts a large stretch of the amino acid sequence.

\section{DISCUSSION}

Two sequence fragments determined in the same $B$. subtilis strain $(168, \operatorname{tr} p C 2)$ overlap over more than $10 \mathrm{~kb}$ (this work and Fuma et al., 1993). Twenty-nine discrepancies were observed between the two sequences. A third sequence obtained from another strain (Cosmina $e t$ al., 1993) was used to resolve the conflicts and allowed the estimation of the total error rate in the finished sequences, corresponding to $0.02 \%$ for our sequence (X72672) and
$0 \cdot 27 \%$ for D13262 (Fuma et al., 1993); these numbers included random and systematic errors. The latter kind of error, corresponding to sequence-specific discrepancies associated with compressions on our gels, can be discarded. Thus, the estimates of the random error rate for X72672 and D13262 are $0.01 \%$ and $0 \cdot 13 \%$, respectively.

According to the theoretical work of Churchill \& Waterman (1992), the $0 \cdot 13 \%$ random error rate, obtained for D13262, is in the range of what is expected for a shotgun approach (assuming a $10^{-2}$ error rate for gel reading and a redundancy rate of 6 ). On the other hand, the $0.01 \%$ random error rate, which we obtained with a coverage of 5.5 , is one order of magnitude below this standard. Manual film readings have been shown to give the lowest error rate $\left(5 \times 10^{-3}\right.$; Khurshid \& Beck, 1993) but it seems that ours were done with a much higher degree of accuracy, very likely reaching a reading error rate much lower than $10^{-3}$. Indeed, we used a manual reader (see Methods) equipped with a speaker that echoes back bases when entering them, thus allowing us to easily detect misreadings. In addition, almost all the sequences were read independently by two investigators.

Only four discrepancies in X70356, corresponding either to true errors or to natural variations, are associated with compressions detected in our gels. This is a small value in comparison to the 15 discrepancies found in D13262 also shown to correspond to compressions in our gels. This difference is most likely due to the sequencing method used by the Japanese team (Fuma et al., 1993). They certainly must have had difficulties detecting compressions, and using a 'mono-protocol' approach, without any change in the sequencing reactions or electrophoresis, they were unable to resolve them. Thus, when assembling sequences in a shotgun approach, systematic false readings of the same area in different templates may result in misconstruction of the finished sequence, giving errors in this region. Tools to resolve the compressions are available, but the limiting factor is their detection. A manual reading of gels by an expert gives better results than automatic film or fluorescence readers, but at a much higher cost. Thus, compressions should be well characterized in such a way that defined rules could be included in algorithms to achieve automatic detection. On the other hand, as systematic errors are assumed to be sequence-specific, one can expect to be able to detect such error-prone regions throughout the analysis of the local sequence properties. Characterization of their folding tendency is the first step along this route. To complete this approach, a database of sequencing data (with regard to compressions and their readings) would be very useful.

DNA sequencing is still a very expensive technique and one way to reduce its cost would be to decrease the redundancy rate. We have recently proposed a new strategy to achieve this goal with large fragments ( $>100 \mathrm{~kb}$, Denizot \& Guenoche, unpublished data). Computer simulations showed that all contigs can be ordered with a mean coverage between 2 and 3 . The gaps between contigs are easily determined by a directed approach. Each base is sequenced on both strands and areas 
showing any sequence discrepancies are sequenced once again. In the case of a pure random error, with an error reading rate $(r)$ of $10^{-2}$, the likelihood of obtaining an error at the same position in the third sequence is given by $r^{2}=10^{-4}$. An on-line analysis of the assembled sequence with the modified RECSTA program will be helpful in detecting frameshift errors. Since less work is needed to cover rapidly the total DNA fragment and to obtain the sequence roughly at a low random error rate, more effort could be made to detect and resolve systematic errors to obtain a very accurate finished sequence.

\section{ACKNOWLEDGEMENTS}

The authors are grateful to Dr G. Fichant for helpful discussions and to Dr A. Cornish-Bowden for comments on the manuscript. Support was provided by institutional grants from the Centre National de la Recherche Scientifique, the Ministère de l'Education Nationale and the Groupement de Recherche et d'Etudes sur les Génomes.

\section{REFERENCES}

Anagnostopoulos, C., Piggot, P. J. \& Hoch, J. A. (1993). The genetic map of Bacillus subtilis. In Bacillus and Other Gram-Positive Bacteria: Biochemistry, Physiology and Molecular Genetics, pp. 425-461. Edited by A. L. Sonenshein, J. A. Hoch \& R. Losick. Washington, DC: American Society for Microbiology.

Altschul, S. F., Gish, W., Miller, W., Myers, E. W. \& Lipman, D. J. (1990). Basic local alignment search tool. J Mol Biol 215, 403-410.

Barnett, R. S. \& Davidson, J. N. (1989). Coating gel plates. Focus 11, 75.

Chen, W. Q. \& Hunkapiller, T. (1992). Sequence accuracy of large DNA sequencing projects. DNA Seq 2, 335-342.

Churchill, G. A. \& Waterman, M. S. (1992). The accuracy of DNA sequences: estimating sequence quality. Genomics 14, 89-98.

Cosmina, P., Rodriguez, F., de Ferra, F., Grandi, G., Perego, M., Venema, G. \& van Sinderen, D. (1993). Sequence and analysis of the genetic locus responsible for surfactin synthesis in Bacillus subtilis. Mol Microbiol 8, 821-831.

De Araujo Novaes, M. \& Denizot, F. (1993). An automatic approach for DNA sequencing. Biochimie 75, 347-351.

Devereux, J., Haeberli, P. \& Smithies, O. (1984). A comprehensive set of sequence analysis programs for the VAX. Nucleic Acids Res 12, 387-395.

Fichant, G. \& Gautier, C. (1987). Statistical method for predicting protein coding regions in nucleic acid sequences. CABIOS $\mathbf{3}$, 287-295.

Fuma, S., Fujishima, Y., Corbell, N., D'Souza, C., Nakano, M. M., Zuber, P. \& Yamane, K. (1993). Nucleotide sequence of $5^{\prime}$ portion of $\operatorname{srf} A$ that contains the region required for competence establishment in Bacillus subtilis. Nucleic Acids Res 21, 93-97.
Garoff, H. \& Ansorge, W. (1981). Improvements of DNA sequencing. Anal Biochem 115, 450-457.

Howard, J. (1992). Formamide gels: relief from compression artefacts. Comments (USB) 19, 62-63.

Khurshid, F. \& Beck, S. (1993). Error analysis in manual and automated DNA sequencing. Anal Biochem 208, 138-143.

Koop, B. F., Rowan, L., Chen, W. Q., Deshpande, P., Lee, H. \& Hood, L. (1993). Sequence length and error analysis of sequenase and automated Taq cycle sequencing methods. BioTechniques 14, 442-447.

Krawetz, S. A. (1989). Sequence errors described in GenBank: a means to determine the accuracy of DNA sequence interpretation. Nucleic Acids Res 17, 3951-3956.

Kristensen, T., Lopez, S. \& Prydz, H. (1992). An estimate of the sequencing error frequency in the DNA sequence databases. $D N A$ Seq 2, 343-346.

Lang, B. F. \& Burger, G. (1990). A rapid, high resolution DNA sequencing gel system. Anal Biochem 188, 176-180.

Mizusawa, S., Nishimura, S. \& Sela, F. (1986). Improvement of the dideoxy chain termination method of DNA sequencing by use of deoxy-7-deazaguanosine triphosphate in place of dGTP. Nucleic Acids Res 14, 1319-1324.

Radola, B. J. (1980). Ultrathin-layer isoelectric focusing in $50-100 \mu \mathrm{m}$ polyacrylamide gels on silanized glass or polyester films. Electrophoresis 1, 43-56.

Rychlik, W. \& Rhoads, R. E. (1990). A computer program for choosing optimal oligonucleotides for filter hybridization, sequencing, and in vitro amplification of DNA. Nucleic Acids Res 17, 8543-8551.

Salles, C., Creancier, L., Claverys, J. P. \& Mejean, V. (1992). The high level streptomycin gene from Streptococcus pneumoniae is a homolog of the ribosomal protein $\mathrm{S} 12$ gene from Escherichia coli. Nucleic Acids Res 20, 6103.

Sambrook, J., Fritsch, E. F. \& Maniatis, T. (1989). Molecular Cloning: a Laboratory Manual. Cold Spring Harbor, NY: Cold Spring Harbor Laboratory.

Sanger, F., Nicklen, S. \& Coulson, A. R. (1977). DNA sequencing with chain-terminating inhibitors. Proc Natl Acad Sci USA 74, 5463-5467.

Sulston, J. and others (1992). The $C$. elegans genome sequencing project: a beginning. Nature 356, 37-41.

Wilson, R. and others (1994). $2 \cdot 2 \mathrm{Mb}$ of contiguous nucleotide sequence from chromosome III of $C$. elegans. Nature 368, 32-38.

Zimmermann, J., Voss, H., Schwager, C., Stegemann, J., Erfle, H., Stucky, K., Kristensen, T. \& Ansorge, W. (1990). A simplified protocol for fast plasmid DNA sequencing. Nucleic Acids Res 18, 1067.

Received 13 July 1994; revised 19 September 1994; accepted 11 October 1994. 